\title{
A heuristic adaptive discrete differential evolution algorithm for cooperative interference decision
}

\author{
Zhang Zhenhua ${ }^{1}$ \\ ${ }^{1}$ Gansu Normal University for Nationalities, Hezuo, gansu, 747000, China
}

Keywords: Differential evolution algorithm; Mathematical model; Adaptive mechanism; Repair operation

\begin{abstract}
Under the push of information, the traditional operation mode of "platform centric" is changing to the "network centric" cooperative combat mode. At present, with the development of aviation, aerospace, navigation technology, based on the concept of Network Centric Warfare, the derivative of the "space war", "air sea battle" as a new concept, with multiple moving platforms between tasks and information of the coupled coordination problem more and more attention and extensive research. A heuristic adaptive discrete differential evolution algorithm was proposed. The algorithm was experimented and the experimental results show that the novel algorithm is better suited to handle cooperative interference decision.
\end{abstract}

\section{Introduction}

Cooperative operation is a cooperative operation based on Networked command information system in the land, sea, air, sky, electric and magnetic field. Under the condition of information local war, the problem of cooperative command has a decisive effect on the outcome of the coordination. Local war is the struggle between the two sides of information system, it is the confrontation of the platform and the platform. But our arms self-contained, failed to form a command information system integration. Therefore, it is very urgent and important to construct a cooperative control platform.

Multiple Platform Cooperative Jamming Decision making(MPCJD) is how to allocate our UCAV to the enemy target radar emitter discharge jamming, to maximize the overall multi frame UCAV interference, and as far as possible to reduce operating losses, MPCJD problem is a NP-C problem ${ }^{[1,2]}$, the with the increasing complexity of solving the problem size exponentially. With the increase of the number of weapons and targets, the exact algorithm to solve the time will not be accepted.

At present, the research on MPCJD is still rare, the paper proposes an heuristic a Heuristic Self-adaptive Discrete Differential Evolution algorithm (H-SDDE). The H-SDDE algorithm maintains a candidate strategies pool, including four effective CSGSs. In the process of evolution, each CSGS obtains a probability value through learning, the greater the probability that the probability value is selected. In this paper, based on the characteristics of the problem, this paper designs an extended integer coding scheme based on threat degree, and an individual repair operation based on constraint satisfaction.

\section{The Problem modeling}

$$
\begin{aligned}
& p=1-\exp \left(1-\int_{t_{1}}^{t_{2}} r(t) d t\right) \\
& r(t)=\theta_{0.5} / \varphi f_{r} e^{-y_{0}} S_{N}(t) \\
& S(t)=P_{t} G_{t} \sigma \Delta f_{j} / 4 \pi L K \theta_{0.5}^{2} \Delta f_{r} P_{j} G_{j} \bullet\left(\theta^{2}(t) R_{j}^{2}(t) / R^{4}(t)\right) \\
& E S P=1-p=\exp \left(1-\mu_{0} \int_{t_{1}}^{t_{2}} \theta^{2}(t) R_{j}^{2}(t)\right) / R^{4}(t) d t \\
& E N / \mathrm{r}^{2} \leq \varepsilon
\end{aligned}
$$




$$
\begin{aligned}
& E C S=\left\{\begin{array}{lll}
0 & \text { if } & d \succ r \\
\varepsilon r^{2} / d^{2} & \text { else }
\end{array}\right. \\
& E F=\left\{\begin{array}{l}
0 \quad \text { if } R_{-} \text {Fre } \cap J_{-} \text {Fre }=\Phi \\
1 \quad \text { else if } R_{-} \text {Fre } \subseteq J_{-} \text {Fre } \\
\left(R_{-} \text {Fre } \cap R_{-} \text {Fre }\right) / R_{-} \text {Fre else }
\end{array}\right. \\
& E J C=\left\{\begin{array}{l}
0 \quad \text { if } \quad J_{-} J a m S t y \cap R \_B e s J a m S t y=\Phi \\
1-\left(\text { Seq_ } R_{-} \text {Bes__ } 1\right) / 20 \text { else }
\end{array}\right. \\
& \mathrm{a}_{i j}=a_{i j} / \sum_{k=1}^{s} a_{k j},(i, j=1,2, \ldots, s) \\
& W_{i}=\sum_{j=1}^{s} a_{i j} / \sum_{k=1}^{s} \sum_{j=1}^{s} a_{k j}^{-},(i=1,2, \ldots, s) \\
& \text { FITN }^{R_{\mathrm{i}}-J_{\mathrm{i}}}=\left\{\begin{array}{l}
0 \quad \text { if } \exists \mathrm{I}_{1} \in I \text { and } I_{1}=0 \\
I \bullet W^{T} \quad \text { else }
\end{array}\right. \\
& f(\pi)=\operatorname{Max} \sum_{i=1}^{m}\left\{\left[\sum_{j=1}^{n} \operatorname{FITN}^{R_{i}-J_{i}} X_{i j}\right] \bullet\left[1-\prod_{i=1}^{n}\left(1-p_{s}^{R_{I}-J_{i}}\right)^{x_{i j}}\right]\right\} \\
& f_{R_{i}} \subseteq \bigcup_{j=1}^{n} f_{J_{j}} x_{i j}, i=1,2, \ldots, m \\
& J_{\text {jHstyle }} \cap R_{\text {iEstyle }} \neq \Phi \quad \text { if } x_{i j}=1 \\
& \bigcap_{j=1}^{n}\left(J_{j H s t y l e} X_{i j}\right) \subseteq R_{i E s y l e}, i=1,2, \ldots, m
\end{aligned}
$$

In the Formula $1, p$ is the discovery probability of UCAV, $r(t)$ is instantaneous probability density of target radar found our platform, UCAV at $t$ time. In the Formula 2, $\theta_{0.5}$ is the radar antenna half power beam width, $\varphi$ is the radar scanning fan angle, $\mathrm{f}_{r}$ is the radar pulse repetition rate, $y_{0}$ is the detection threshold, $S_{N}$ is a single pulse signal to noise ratio. In the Formula 3, $P_{t}$ is the radar transmitting power, $\mathrm{G}_{t}$ is a radar antenna gain, $\sigma$ is the effective reflection area of target, $\Delta \mathrm{f}_{j}$ is launching UCAV interference frequency, $L$ radar power loss factor, $K$ is Pohl Seidman's constant, $P_{j}$ is the interference of UCAV transmitting power, $G_{j}$ is the antenna main lobe direction on the UCAV gain, $\theta(t)$ is the angle between the cover and the radar platform line and radar and UCAV connection, $R_{j}(t)$ is UCAV to the radar range, $R(t)$ is our cover the distance to the radar platform. In the Formula 4, $\mu_{0}=f_{\mathrm{r}} e^{-y_{0}} P_{t} G_{t} \sigma \Delta f_{j} / 4 \pi \varphi L K \Delta f_{r} P_{j} G_{j} \theta_{0.5}$. In the Formula $5, \varepsilon$ is the minimum energy intensity threshold for UCAV to target radar. When the distance between the UCAV and the target is beyond the interference radius, the interference of the target is 0 , otherwise, it is the inversely proportional to the distance $d^{2}, E N$ is the maximum interference energy intensity of UCAV, $r$ is the effective interference radius. In the Formula 6, ECS is evaluation of cover space. In the Formula 7, EF is evaluation of frequency, $R$ _Fre is the target radar working frequency band, $J$ _Fre is the UCAV interference band. In the Formula 8, EJS is evaluation of jamming style, $J \_J a m S t y$ is a set of interference patterns of UCAV, $R$ _BesJamSty is a collection of the best jamming radar, Seq_ $R$ _Bes is the most effective jamming order of UCAV. Formula 9 and Formula 10 are the calculation of the judgment matrix and the calculation of the relative weight. $\square$ the judgment matrix is as follows: 


$$
A=\left[\begin{array}{cccc}
a_{11} & a_{12} & \ldots & a_{1 s} \\
a_{21} & a_{22} & \ldots & a_{2 s} \\
\ldots & \ldots & \ldots & \ldots \\
a_{s 1} & a_{s 2} & \ldots & a_{s s}
\end{array}\right]
$$

In the judgment matrix, $\mathrm{a}_{i i}=1, \quad a_{i j}=1 / a_{j i}, \quad$ and $\quad a_{i j} \succ 0, s$ is the total number of jamming effectiveness evaluation indicators. In the Formula 11, FITN ${ }^{R_{\mathrm{i}}-J_{\mathrm{i}}}$ indicates the interference effect of $J_{j}$ on $R_{i}$. In the Formula 12, $\pi$ indicates cooperative interference scheme, it is denoted as $\pi=(\pi(1), \pi(2), \circ \circ \circ, \pi(n))$, The position of $\mathrm{j}$ represents the UCAV of $\mathrm{j}$, and the numerical of $\mathrm{j}$ position representation the target radar number, $\mathrm{p}_{s}^{J_{i}-r_{i}}$ is the probability of interference success. Formula 13 indicates that the total working frequency band of UCAV cooperative interference should include the current working frequency band of the target radar, $f_{R_{i}}$ is the current working frequency of the target radar $R_{i}, f_{J_{j}}$ is the entire operating band of the Unmanned aircraft $\mathrm{Jj}$. Formula 14 indicates that the UCAV assigned to the target radar should have an effective jamming pattern against the radar jamming, $J_{\text {jHstyle }}$ indicates that $J_{j}$ has a set of interference patterns, $R_{\text {iEstyle }}$ is the best interference pattern set for the target radar $R_{i}$. Formula 15 indicates cooperative jamming is needed to work together in an interference pattern.

\section{Heuristic adaptive discrete differential evolution algorithm}

\subsection{Coding scheme}

This paper proposed an extended integer encoding scheme based on threat degree. when $m>n$, select a higher degree of $n$ radar threat, the same threat degree according to the target number of selected from small to large, then according to the radar threat degree are numbered from 1 to $n$, thus forming integer encoding scheme from 1 to $n$. When $m<n$, according to the threat from large to small target radar from 1 to $m$ number. At last, the target radar number is extended. The code is 1 to $n$. As shown in table 1 .

Table 1 Extended integer coding scheme based on threat degree

\begin{tabular}{|c|c|c|c|c|c|c|c|c|c|c|}
\hline 1 & 2 & $\ldots$ & $\ldots$ & $\ldots$ & $\ldots$ & $\ldots$ & $\ldots$ & $\ldots$ & $\ldots$ & $n$ \\
\hline 1 & 2 & $\ldots$ & $\mathrm{m}$ & 1 & 2 & $\ldots$ & $m$ & 1 & $\ldots$ & $s$ \\
\hline
\end{tabular}

\subsection{Adaptive mechanism}

In this algorithm, the value of the control parameter CR is largely dependent on the practical problems, and the good CR parameters can make the algorithm have better performance. Therefore, according to the CR value, the paper has better adaptive adjustment of the strategy parameters. For the $q$ of CSGS, define the corresponding mean value $C R_{m q}$, the initial value is 0.5 . The mean vector of the CR parameters of the 4 kinds of CSGS is $C R_{\mathrm{m}}=\left(C R_{m 1}, C R_{m 2}, \ldots, C R_{m Q}\right)$, and the Gauss distribution $N\left(C R_{m q}, 0.1\right)$ is used to generate the CR value for each individual of the CSGS.

\subsection{Individual repair}

In order to improve the solving speed, this paper designs a heuristic individual adjustment operation based on the threat degree according to the practical problem:

Assuming that there are 4 UCAVs and 4 radar targets, the assumption is that the threat of the target is obtained by the reconnaissance.

THREAT $=[0.85,0.5,0.95,0.3]$, equipment jamming effectiveness evaluation matrix:

$$
\text { FITN }=\left[\begin{array}{cclc}
0.7 & 0.6 & 0.45 & 0.8 \\
0.5 & 0.7 & 0.4 & 0.9 \\
0.6 & 0.5 & 0.75 & 0.7 \\
0.3 & 0.4 & 0.2 & 0.1
\end{array}\right]
$$


Assuming $U$ vector: $U=\underline{1} \underline{4} \underline{2} \underline{3}$, at first, select a number of bits in accordance with a certain probability, assuming that selected 1 bit, 2 bit, 3 bit, and then sort for $R_{1}, R_{2}, R_{4}$ according to the threat degree of the target radar. Select $R_{1}, F_{1 T N}$ is the largest, assign $J_{1}$ to $R_{1}$; Select $R_{2}, F_{1 T N}$ is the largest, assign $J_{2}$ to $R_{2}$; Select $R_{4}$, FITN $_{24}>$ FITN $_{14}>$ FITN $_{34}$, but $J_{1}$ and $J_{2}$ have been allocated, so the distribution of $J_{3}$ to $R_{4}$; For the target radar that is not selected, the random selection is not assigned bit to fill; therefore, the final $U$ vector is adjusted to $U=\underline{1} \underline{2} \underline{4}$.

In order to deal with the constraints, this paper designs individual repair operation based on the constraint satisfaction according to the actual problem, the example described as follows: suppose there are 10 UCAVs and 6 radar targets, radar target according to the threat sequencing for (3, 2, 5, $1,4,6)$, according to the extended integer encoding scheme, 6 goals is extended to 10 goals, including the radar target 7, 8, 9, 10 corresponding to 3, 2, 5, 1 .

Assume that the new individual is $(1,4,8,2,10,3,9,5,6,7)$, firstly, decoding operation to the $U$ vector into $(1,4,2,2,1,3,5,5,6,3)$, then check the $U$ vector by using the constraint condition (Formula 13) (Formula 15), $x$ bits remain unchanged in accordance with the constraint conditions and the fitness is greater than 0.5 , which the bits do not conform to the constraints and the fitness is low (10- $x$ ) and random exchange position.

\section{Simulation experiment}

\subsection{Experiment environment and parameter setting}

The simulation experiment is carried out on the platform of a cooperative electronic countermeasure simulation experiment, which includes the scene setup module, the cooperative reconnaissance module and the cooperative jamming module. The cooperative jamming is based on Cooperative reconnaissance and data sharing. Twelve different scale test cases are set according to the UCAV number and the number of different target radar (as shown in Table 2), Twelve examples are used to test the proposed H-SDDE algorithm and IIGA ${ }^{[3]}, \mathrm{WRGA}^{[4]}, \mathrm{ACO}^{[5]}$, SA-DPSO ${ }^{[6]}$. The performance test of each simulation test algorithm is run independently 50 times, and the statistical results are compared and analyzed.

H-SDDE algorithm parameters: NP is equal to the number of UCAV; $Z$ is equal to the number of radar target, the initial $p_{q}=0.25(q=1,2, \ldots, 4)$; the initial $C R_{m q}=0.5$; according to the preliminary experimental experience, $\mathrm{LP}=5$, and every 50 generation strategy selection matrix initialization time; number of fitness evaluations assessment (NFE) is 100000 . IIGA, WRGA, ACO; SA-DPSO, NFE and other parameters in running times were set up according to the parameters in the original document; for programming convenience, this thesis takes the reciprocal of the objective function $(1 / f(\pi))$ as fitness function.

Table 2 Algorithm comparison

\begin{tabular}{|c|c|c|c|c|c|c|c|c|}
\hline Algorithm & \multicolumn{2}{|c|}{ Swap } & \multicolumn{2}{c|}{ Reverse } & \multicolumn{2}{c|}{ DDE } & \multicolumn{2}{c|}{ H-SDDE } \\
\hline Example & Mean & Std & Mean & Std & Mean & Std & Mean & Std \\
\hline $10 * 5$ & $1.1 * 10^{-1}$ & $7.6 * 10^{-4}$ & $1.1 * 10^{-1}$ & $1.5 * 10^{-3}$ & $1.1 * 10^{-1}$ & $1.0 * 10^{-4}$ & $1.1 * 10^{-1}$ & 0 \\
\hline $10 * 10$ & $1.2 * 10^{-1}$ & $1.8 * 10^{-3}$ & $1.2 * 10^{-1}$ & $3.0 * 10^{-3}$ & $1.2 * 10^{-1}$ & 0 & $1.2 * 10^{-1}$ & 0 \\
\hline $30 * 10$ & $3.6 * 10^{-2}$ & $2.6 * 10^{-4}$ & $3.6 * 10^{-2}$ & $4.2 * 10^{-4}$ & $3.5 * 10^{-2}$ & $2.1 * 10^{-4}$ & $3.5 * 10^{-2}$ & 0 \\
\hline $30 * 20$ & $3.6 * 10^{-2}$ & $1.4 * 10^{-4}$ & $3.7 * 10^{-2}$ & $3.3 * 10^{-4}$ & $3.6 * 10^{-2}$ & $3.7 * 10^{-4}$ & $3.6 * 10^{-2}$ & 0 \\
\hline $50 * 30$ & $2.1 * 10^{-2}$ & $1.4 * 10^{-4}$ & $2.2 * 10^{-2}$ & $1.6 * 10^{-4}$ & $2.1 * 10^{-2}$ & $5.3 * 10^{-5}$ & $2.1 * 10^{-2}$ & $1.7 * 10^{-5}$ \\
\hline $50 * 50$ & $2.1 * 10^{-2}$ & $1.1 * 10^{-4}$ & $2.1 * 10^{-2}$ & $2.3 * 10^{-4}$ & $2.1 * 10^{-2}$ & $2.2 * 10^{-5}$ & $2.1 * 10^{-2}$ & $1.4 * 10^{-5}$ \\
\hline
\end{tabular}

In order to compare the solving speed of the proposed algorithm with other algorithm, Figure 1 plots fitness average with the evaluation times change of convergence characteristic curve in two typical test examples of $10 * 10$ and $50 * 50$ of the Algorithm in table 2 (the convergence curve of other test instances are similar). In order to observe conveniently, the natural logarithm of the number of evaluation is used as the horizontal coordinate. It can be seen in Figure 1: firstly, in the $10 * 10$ and 50*50 two test examples, H-SDDE can converge to the optimal solution in a limited number of assessment, the comparison of algorithms can not converge or converge to the local 
optimal solution. For example, ACO and WRGA converge to local optimal solution in the $10 * 10$ instance, IIGA and SA-DPSO can not converge in a limited number of assessment, ACO converges to the local optimal solution in the 50*50 examples, IIGA, WRGA and SA-DPSO can not converge. This shows that H-SDDE has better global optimization ability. Secondly, look from the quality of the solution, the quality of the H-SDDE solution is better than other algorithms to solve the quality of the solution in a limited number of times.
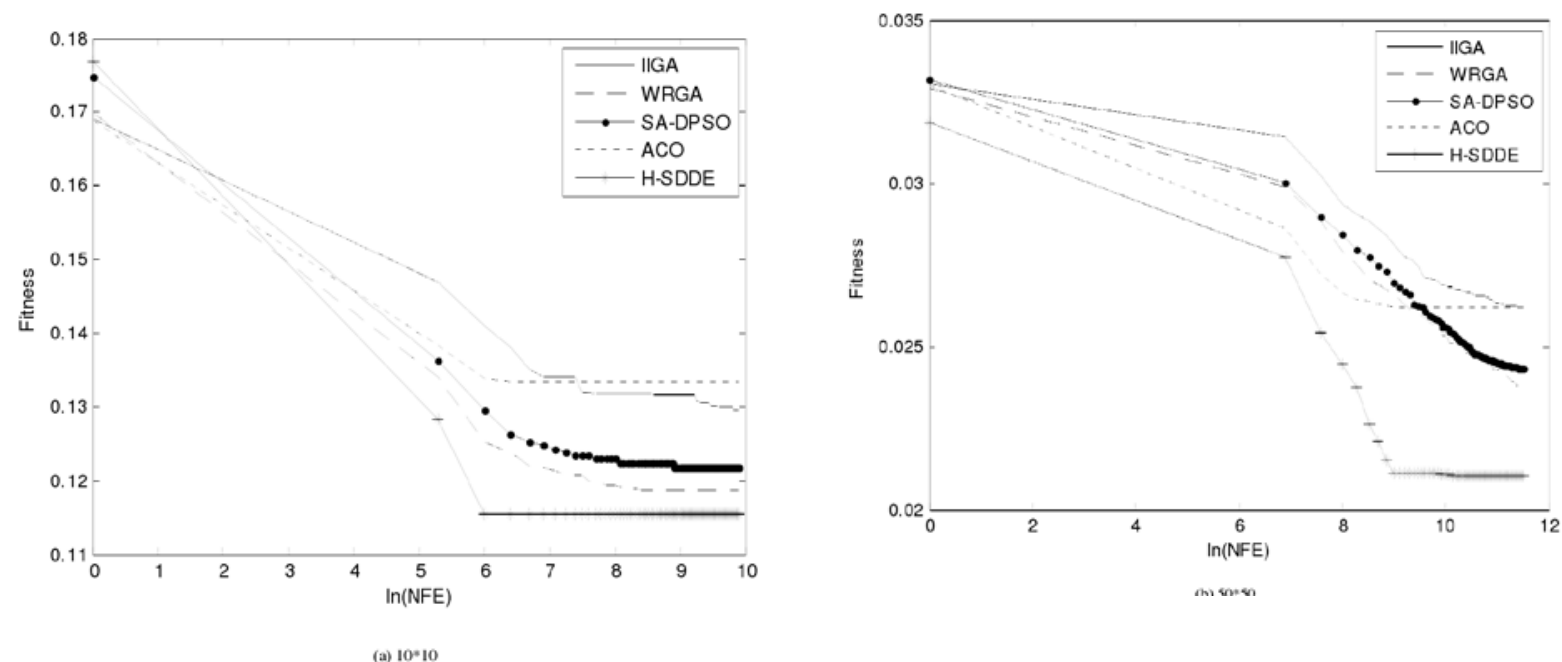

Fig. 4 The convergence curves of H-SDDE and other algorithms

\section{Conclusions}

Aiming at modern combat environment in multi UCAV cooperation against multiple threat radar mission planning a comprehensive analysis of the problems of military decision making through operations research, decision index of interference suppression probability assessment 4 assessment, and gives the quantitative method of evaluation index. Then, the weight calculation method based on AHP is proposed, and the calculation steps of this method are described in detail. H-SDDE algorithm is proposed in this paper. An extended integer coding scheme based on threat degree is proposed. Simulation results show that CSGS and its parameter adaptive learning process, individual adjustment heuristic repair operation can significantly enhance the quality of solution and the speed of algorithm, it can significantly improve the overall operational performance of cooperative jamming.

\section{References}

[1] Xing Wenxun, Xie Venus. Modern optimization calculation method[M]. 2005:2-46.

[2] S. P. Lloyd, H. S. Witsenhausen. Weapons allocation is NP-complete. The 1986 Summer Conference on Simulation[C], 1986:1054-1058.

[3] X. F. Zhai, Y. Zhuang. IIGA based algorithm for cooperative jamming resource allocation. Asia Pacific Conference on Postgraduate Research[C], 2009:368-371.

[4] Y. Xue, Y. Zhuang, T. Q. Ni, S. R. Ni. One improved genetic algorithm applied in the problem of dynamic jamming resource scheduling with multi-objective and multi-constraint. IEEE Fifth international conference on Bio-inspored Computing: Theories and Applications[C], 2010:708-712.

[5] Luo Delin, Duan seashore, Wu Shun, Li Maoqing. Study on the decision making of cooperative multi target attack based on heuristic ant colony algorithm. Journal of Aeronautical Sciences, [J], 2006, 27 (06): 1166-1170.

[6] Li Yan, Dong Yuna. The fire distribution of Cooperative Air Combat Based on SA-DPSO hybrid optimization algorithm. Journal of Aeronautics and the [J], 2010, 31 (03): 626-631. 